\title{
Does Short Selling Improve Corporate Social Responsibility?
}

\author{
Renshu Yuan ${ }^{1,2^{*}}$ Jiguo Yang' ${ }^{1}$ Lei Gao $^{3}$ \\ ${ }^{1}$ Economics School, Xiamen University, Xiamen, Fujian 361005 \\ ${ }^{2}$ School of Economics and Management, Guizhou Normal University, Guiyang, \\ Guizhou 550025 \\ ${ }^{3}$ College of Business, Iowa State University, Ames Iowa IA50011
}

\begin{abstract}
Using difference-in-difference (DID) model, this paper studies firms' incentives to engage in corporate social responsibility (CSR) activities based on the quasi-natural experiment. We find that firms experiencing an exogenous increase in their exposure to short sales significantly raise their CSR activities. The results are stronger for firms that are more short sold, higher ratio of the largest shareholder, internal control more effectively. Our evidence is consistent with the argument of CSR being a signaling device used by managers to reduce information asymmetry.
\end{abstract}

Keywords

Corporate Social Responsibility; Margin trading, Signaling; Short sale

\section{卖空机制促进了企业社会责任吗？ 袁仁书 ${ }^{1,2 *}$ 杨继国 ${ }^{1}$ 高磊 $^{3}$ \\ 1厦门大学经济学院, 厦门 361005, 中国 \\ 2 贵州师范大学经济与管理学院, 贵阳 550025, 中国 \\ 3 爱荷华州立大学商学院, Ames IA50011, 美国}

摘要: 本文利用中国 $\mathrm{A}$ 股市场实施融资融券规则作为外生的准自然实验, 基于企业层面数 据, 运用双重差分（DID）模型检验企业社会责任活动信号动机。研究发现, 融资融券规则 实施使得作为信号来缓解信息不对称问题的企业社会责任活动显著增加。此外，增加效应在 融券卖空数量较多、第一大股东持股比例较高和内部控制较有效的公司中更显著。研究结果 为制定企业社会责任政策提供了参考依据，也对投资者正确估值公司具有指导意义。

关键词：企业社会责任；融资融券；信号机制；准自然实验

\section{1. 引言}

企业社会责任实践新形势引起了 来自经济学、金融学和管理学等学科
的研究者广泛关注，对企业社会责任 有关问题进行了深入研究。已有文献 主要集中于研究企业履行社会责任的 影响方面。概括起来主要有两种观 
点: 一种观点认为, 企业开展社会责 任活动, 可以通过解决不同利益相关 者之间的矛盾等建立竞争优势而获取 产品差异带来的溢价, 为公司创造价 值（Benabou 和 Tirole，2010）。另 一种观点认为, 企业履行社会责任会 造成企业资源浪费，增加企业经营成 本, 是以牺牲股东利益作为代价来为 管理者自身谋取利益, 会损害公司价 值（Friedman，1970）。

本文研究融资融券规则实施对企 业社会责任活动的政策影响。中国 A 股市场融资融券规制实施为本文的研 究提供了一个准自然实验环境, 也为 本文检验规制变化对企业社会责任活 动的影响提供了难得的机会。本文采 用双重差分 (DID) 模型研究企业社会 责任活动动因, 能相对有效解决已有 研究中存在的内生性问题, 有利于得 到可靠的研究结论。本文的主要贡献 一是从企业社会责任活动的动因方面 研究企业社会责任, 使得动因研究文 献得到丰富; 二是在研究中使用双重 差分模型, 能有效缓解模型中的内生 性问题，使得实证结果更为可靠。

\section{2. 文献综述与研究假设}

\section{1 文献综述}

当公司基本面因素恶化且被投资 者获知时投资者就会卖空公司股票。 另一方面，趋势投资者也会在股票熊 市运行时卖空公司股票。对股票市场 的研究发现, 股票市场放开卖空交易 限制会导致对公司股票卖空增多

(Diether 等, 2009 ; 李志生等, 2015 ），经理们担心卖空引起其他不 利反应会刺激他们释放有关公司真实 情况的信号 (陈国进和张贻军, 2009; Goldstein 等，2013）。

当公司受到释放信号激励，就会 利用诸如分红和打广告 (Chemmanur 和 Yan, 2009) 等活动来对外界释放
信号传递有关公司的真实情况等信 息。Fombrun 和 Shanley ( 1990 ) 认 为, 企业可以通过社会责任行为给企 业带来声誉, 进而向外界传达有关企 业自身经营质量的信号。

现存对企业社会责任研究的文献 大部分关注的是企业社会责任行为和 公司财务绩效方面的联系 (Flammer, 2015）。Hong等（2012）发现公司在 财务宽松的情况下才会进行企业社会 责任方面的投资。Lys（2015）发现, 公司预期其未来财务业绩变好也会进 行社会责任投资，而外界能够从公司 社会责任行为推断公司未来财务业绩 会变好。

\section{2 研究假设}

如文献部分所述，当公司股票卖 空限制减少或解除后，信息不对称问 题会激励公司经理释放信号将自己公 司从基本面恶化而被卖空的公司中区 分出来, 向外界证明本公司基本面情 况并未恶化, 试图阻止或减少卖空行 为。在中国 $\mathrm{A}$ 股市场融资融券试点开 始后, 列入试点名单的公司相对于未 列入试点名单的公司的卖空限制减少 将会引起卖空行为的增加, 对公司股 价施加下跌的压力。公司经理有强烈 的激励去向外界释放信号。因此, 本 文第一个假设为:

$\mathrm{H} 1$ ：列入融资融券名单的公司会 比未列入融资融券名单的公司显著增 加其企业社会责任活动。

当公司列入融资融券试点名单之 后，不同的公司面临的卖空压力必然 会存在差别。因此, 本文的第二个假 设为:

$\mathrm{H} 2$ : 如果被融券卖空股票数量越 多, 则增加企业社会责任活动的效应 越显著。

公司的内部控制越有效，公司的 经营目标越有实现的保障。因此, 本 文提出第三个假设。 
H3：列入融资融券试点名单的公 司, 如果内部控制没有缺陷, 则面临 卖空压力时增加社会责任活动的效应 更显著。

公司股权集中, 大股东拥有更多 决策话语权。当公司第一大股东持股 比例越高, 在融券卖空的压力下, 公 司在企业社会责任方面的投资越积 极。因此, 本文提出第四个假设。

$\mathrm{H}_{4}$ ：如果第一大股东持股比例越 高, 则面临卖空压力时公司增加企业 社会责任活动的效应越显著。

\section{3. 研究设计}

本文在中国 $\mathrm{A}$ 股市场实施融资融 券规则所创造的准自然实验环境中应 用双重差分（DID）模型来对以上假设 进行实证检验, 分析融资融券规则对 企业社会责任活动的影响及其机制。

中国在 $\mathrm{A}$ 股市场实施融资融券规则的 根本目的在于丰富市场投资手段和发 现公司价值，这一政策是外生于企业 社会责任活动的, 这使得融资融券规 则实施为本文提供了一个研究的准自 然实验环境

本文的样本区间为 2009-2014 年, 以列入融资融券试点名单的样本 公司作为处理组, 未列入试点名单的 样本公司作为控制组, 以样本公司列 入融资融券试点名单的时间作为政策 实施时间。回归估计时, 本文首先比 较列入融资融券试点名单公司和未列 入融资融券试点名单公司在政策实施 前企业社会责任活动的差异, 再比较 列入融资融券试点名单公司和未列入 融资融券试点名单公司在政策实施后 企业社会责任活动的差异，完成一阶 差分过程, 然后对这两个差异进行二 阶差分，所得结果即是融资融券规制 实施对企业社会责任活动的政策效 应。为完善研究结论, 本文还从卖空 强度、内部控制和股权集中度三个方 面对研究样本分组进行异质性分析。

\section{1 模型与变量}

本文设定如下模型:

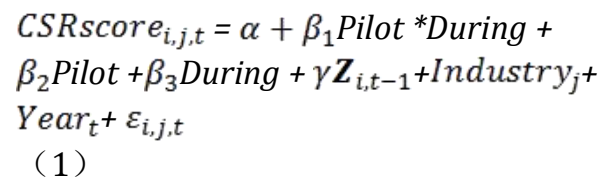

其中, $i$ 表示公司, $j$ 表示行业, $t$ 表示时 间, CSR score $e_{i, j, t}$ 表示某一个公司在给 定行业和给定年份的润灵环球企业社 会责任 (CSR) 评分。Pilot 为二值变 量, 当公司被列入融资融券试点名单 时为 1 , 表示样本公司为处理组; 公司 未进入融资融券名单为Pilot 值为 0 , 表 示样本公司为控制组。During也是二 值变量, 当处于试点期其值为 1 , 否则 为 0 。因此, 交乘项 Pilot ${ }^{*}$ During 的系 数则是我们感兴趣的双重差分（DID） 估计量, 度量的是列入融资融券试点 名单的公司相对未列入试点名单的公 司政策实施前后 CSR 评分的变化, 该 系数反映了融资融券规则实施对处理 组公司社会责任活动的政策效果。本 文在分析中还引入了一系列控制变 量, 以向量 $\mathbf{Z}$ 表示, 包括总资产Tobin $Q$ 和 leverage、现金流、总资产回报 率。Industry 和 Year 是行业固定效应 和年度固定效应。

\section{2 样本、数据选取及预处理}

\section{1、企业社会责任活动数据}

本文采用润灵环球（RKS）企业 社会责任评级机构对中国A股上市公司 社会责任报告评分值来衡量企业社会 责任活动情况。润灵环球（RKS）采 用指数法衡量企业社会责任报告里反 映的社会责任履行和披露情况, 其评 价体系采用独创的MCTi评级体系, 依 据结构化的评分方法从完整性、内 容、技术和产业四个方面展开。在该 评价体系中, 公司得分越高其社会责 任综合表现越优秀。本文搜集了 
2009-2014年企业社会责任数据, 共 11341 个观察值。

\section{2、融资融券数据}

在中国 $\mathrm{A}$ 股市场上实施融资融券 规则经历了一个循序渐进的过程。 2010 年 3 月至 2014 年 9 月先后四次调 整标的股票, 相关数据来自沪、深交 易所。有关融资融券数据、财务数 据、公司治理数据和公司内部控制数 据均来自国泰安数据库 (CSMAR)。

\section{4. 实证结果分析与讨论}

\section{1 基本分析}

1、描述性统计和特征变量比较

为了分析融资融券规则实施对公 司承担企业社会责任活动的影响, 满 足双重差分 (DID) 模型的要求, 本文 首先检查处理组和控制组样本公司各 变量在融资融券规则实施前是否具有 可比性。其次, 对样本中处理组和对 照组进行对比分析。经分析可以看 出, 在融资融券规则实施之前, 控制 组的 CSR 评分均值为 31.956 , 处理组 的 CSR 评分均值为 31.923, 二者相差 仅仅 0.033 分, 无显著差异。这表明在 融资融券规则实施之前, 控制组和处 理组样本公司的社会责任活动处于相 似水平。因篇幅所限, 分析数据略。

\section{2、回归分析}

为避免影响公司承担社会责任的 潜在因素干扰分析结论, 本文通过控 制其他因素进行双重差分（DID）分

表 1 双重差分 (DID) 分析

\begin{tabular}{|l|c|c|c|c|}
\hline VARIABLES & $(1)$ & $(2)$ & $(3)$ & $(4)$ \\
\hline Pilot*during & $2.488^{* * *}$ & $2.624^{* * *}$ & $2.592^{* * *}$ & $2.455^{* * *}$ \\
& $(0.008)$ & $(0.005)$ & $(0.004)$ & $(0.000)$ \\
\hline Pilot & -0.033 & -0.449 & -0.433 & \\
& $(0.960)$ & $(0.512)$ & $(0.520)$ & \\
\hline During & $2.348^{* * *}$ & $2.368^{* * *}$ & $\begin{array}{c}2.356^{* * *} \\
(6.87 \mathrm{e}-\end{array}$ & $\begin{array}{c}2.245^{* * *} \\
(0.000)\end{array}$ \\
& $(1.62 \mathrm{e}-05)$ & $(1.23 \mathrm{e}-05)$ & $06)$ & Yes \\
\hline $\begin{array}{l}\text { Control } \\
\text { variable }\end{array}$ & No & Yes & Yes & Yes \\
\hline Time FE & No & No & No & Yes \\
\hline Industry FE & No & No & Yes & 626 \\
\hline Observations & 662 & 626 & 626 & 0.738 \\
\hline R-squared & 0.091 & 0.127 & 0.213 & \\
\hline \\
注: $* * * \mathrm{p}<0.01, * * \mathrm{p}<0.05, * \mathrm{p}<0.1$ 。括号内为P值。 & \\
\end{tabular}

析, 来检验融资融券规则实施对公司 社会责任活动产生怎样的影响。分析 结果如表 1 所示。其中模型 (1) 未进 行变量控制。可以看到双重差分估计 量 Pilot*during 的系数为 2.488 。模型

(2) 引入控制变量进行双重差分分 析, Pilot*during 的系数为 2.642 , 且 在统计上达到 $1 \%$ 水平显著。说明在控 制了潜在的可能影响公司社会责任活 动的因素之后, 本文的主要分析结论 仍然成立。模型 (3) 和 (4) 考虑了 时间和行业固定效应, Pilot*during 的 系数为 2.592 和 2.455 , 均达到 $1 \%$ 水平 显著, 主要结论仍然成立。

通过双重差分（DID）分析检验, 本文的研究假设 $\mathrm{H}_{1}$ 不能拒绝, 说明融 资融券规则的实施使处理组公司确实 比控制组公司显著增加了企业社会责 任活动。背后的原因在于企业社会责 任活动的信号机制, 也即公司为解决 实施融资融券规则导致的公司与投资 者之间的信息不对称问题而采用社会 责任活动来释放信号这一机制。

\section{5. 异质性分析}

经过以上分析, 本文核心假设得 到验证。本文想进一步追问, 融资融 券规则实施对公司开展社会责任活动 的影响存在异质性影响吗? 具体的影 响因素有哪些呢?

\section{1 释放信号激励强度的异质性分析}

本文对列入融资融券试点 名单的公司按卖空数量中位数 划分为被卖空多和被卖空少的 两组子样本, 检验当激励程度 不同时融资融券规则的实施对 公司社会责任活动的影响有无 差异。分析结果如表 2 所示。 模型 (1) 和 (2) 是对融券卖 空数量小于中值的子样本双重 差分分析, 模型 (3) 和 (4) 是对融券卖空数量大于中值的 
子样本双重差分分析, 分别对应释放 信号激励的强和弱。模型（1）和

(2) 中, Pilot*during 系数均不显 著, 而在模型（3）和（4）, Pilot*during 系数分别为 2.629 和 2.78 , 达到 $10 \%$ 显著水平。分析 结果显示, 融券卖空数量多的公 司比融券卖空数量少的公司增加 了社会责任活动, 假设 $\mathrm{H}_{2}$ 得到 验证。即融资融券规则实施后, 被卖空股票数量多的公司由于卖 空的压力更大, 公司释放信号的 激励更强烈, 从而增加社会责任 活动的效应更显著。
则实施对公司开展社会责任活动受到 公司内部控制是否有效的异质性影 响, 内部控制无缺陷的公司显著高于

表 3 内部控制是否有缺陷的异质性分析

\begin{tabular}{|l|c|c|c|c}
\hline $\begin{array}{c}\text { 公司内部控制 } \\
\text { 是否有缺陷 }\end{array}$ & $\begin{array}{c}(1) \\
\text { 是 }\end{array}$ & $\begin{array}{c}(2) \\
\text { 是 }\end{array}$ & $\begin{array}{c}(3) \\
\text { 否 }\end{array}$ & $\begin{array}{c}(4) \\
\text { 否 }\end{array}$ \\
\hline Pilot*during & $2.910^{*}$ & 2.740 & $2.239^{* *}$ & $2.402^{* *}$ \\
& $(0.079)$ & $(0.110)$ & $(0.047)$ & $(0.032)$ \\
\hline Pilot & -0.732 & -0.124 & 0.378 & -0.444 \\
& $(0.532)$ & $(0.920)$ & $(0.634)$ & $(0.590)$ \\
\hline During & $2.119^{* *}$ & $2.494^{* *}$ & $2.485^{* * *}$ & $2.390^{* * *}$ \\
& $(0.027)$ & $(0.011)$ & $(0.001)$ & $(0.002)$ \\
\hline Control varible & No & Yes & No & Yes \\
\hline Observations & 246 & 224 & 416 & 402 \\
\hline R-squared & 0.075 & 0.137 & 0.105 & 0.145 \\
\hline
\end{tabular}

内部控制有缺陷的公司。背后的原因

表 2 释放信号激励强度的异质性分析

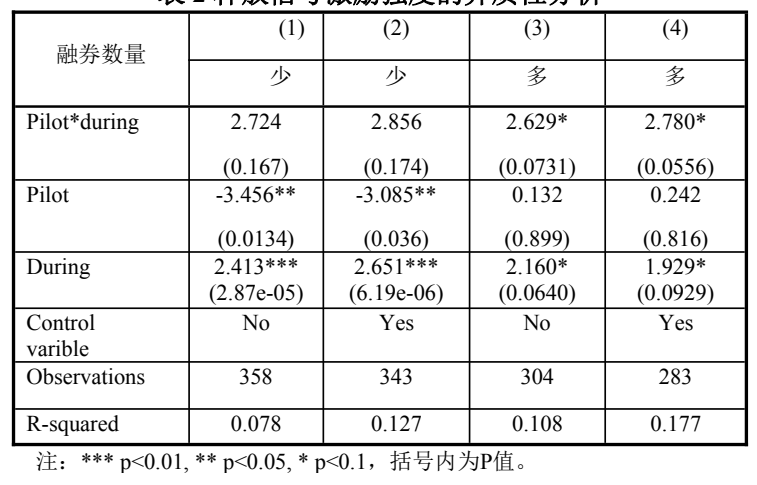

\section{2 公司内部控制异质性分析}

为对假设 $\mathrm{H}_{3}$ 进行检验，本文对试 点公司按公司内部控制有效性的差异 划分为内部控制有缺陷和无缺陷两组 子样本进行双重差分分析。

分析结果如表 3 所示。模型 (1) 和 (2) 是对公司内部控制有缺陷的子 样本双重差分分析, 模型 (3) 和

（4）是对公司内部控制无缺陷的子样 本双重差分分析。模型 (3) 和模型

(4) 的 Pilot*during 系数为 2.239 和 2.402 , 在 $5 \%$ 水平上显著, 而模型 (1) 和模型 (2) 中 Pilot*during 系数 均不显著。分析结果说明融资融券规
可能在于公司内部控制无缺陷能够保 证公司经理更好地执行有利于公司的 决策。

\section{3 股权集中度异质性分析}

股权集中度用第一大股东持股比 例来衡量，反映了公司大股东的话语 权状况，第一大股东持股比例高的公 司开展有利于公司利益的活动易于得 到大股东的支持。本文按公司第一大 股东持股比例中值将研究样本划分为 持股比例高、低两组子样本, 然后进 行双重差分检验。分析结果如表 4 所 示。模型（1）和（2）是对公司第一

表4股权集中度的异质性分析

\begin{tabular}{|l|c|c|c|c|}
\hline $\begin{array}{c}\text { 第一大股东 } \\
\text { 持股比例 }\end{array}$ & $\begin{array}{c}(1) \\
\text { 低 }\end{array}$ & $\begin{array}{c}(2) \\
\text { 低 }\end{array}$ & $\begin{array}{c}(3) \\
\text { 高 }\end{array}$ & $\begin{array}{c}(4) \\
\text { 高 }\end{array}$ \\
\hline Pilot*during & 2.180 & 2.179 & $2.772^{* *}$ & $2.987^{* *}$ \\
& $(0.108)$ & $(0.110)$ & $(0.033)$ & $(0.023)$ \\
\hline Pilot & 0.508 & 0.102 & -0.565 & -0.929 \\
& $(0.596)$ & $(0.919)$ & $(0.538)$ & $(0.327)$ \\
\hline During & $2.243^{* * *}$ & $2.364^{* * *}$ & $2.456^{* * *}$ & $2.445^{* * *}$ \\
& $(0.004)$ & $(0.003)$ & $(0.001)$ & $(0.001)$ \\
\hline $\begin{array}{l}\text { Control } \\
\text { variable }\end{array}$ & No & Yes & No & Yes \\
\hline $\begin{array}{l}\text { Observation } \\
\text { s }\end{array}$ & 330 & 323 & 332 & 303 \\
\hline R-squared & 0.084 & 0.120 & 0.101 & 0.149 \\
\hline
\end{tabular}


大股东持股比例低的子样本双重差分 分析，模型（3）和 (4) 是对公司第 一大股东持股比例高的子样本双重差 分分析。从回归结果可以看出, 第一 大股东持股比例较高组的 Pilot*during 系数为 2.772 和 2.987 , 在 $5 \%$ 的水平上 显著; 当公司第一大股东持股比例较 低时, Pilot*during 系数均不显著。说 明当第一大股东持股比例较高对公司 在融资融券规则实施时开展社会责任 活动时具有显著增加效应, 假设 $\mathrm{H}_{4}$ 得 到验证。背后的原因可能是, 解决融 资融券规则实施后导致的信息不对称 问题符合公司利益，第一大股东持股 比例较高的公司由于大股东拥有更多 话语权更易于执行这一正确决策。

综上所述, 释放信号激励强度、 公司内部控制是否有缺陷和股权集中 度的异质性分析结果进一步验证了本 文的预期并支持了主要分析结论。由 于企业社会责任活动具有信号机制作 用, 融资融券规则实施对企业开展社 会责任活动具有显著影响效果, 而且 这一政策效应受到卖空数量增加形成 的信号释放激励、公司内部控制有效 和第一大股东持股比例高的异质性影 响。

\section{6. 研究结论和进一步说明}

本文检验了企业社会责任活动背 后的信号机制。利用双重差分模型分 析发现, 融资融券规则实施后列入融 资融券试点名单中的公司相对于非试 点公司在试点期间显著增加了它们的 企业社会责任活动, 背后的原因在于 融资融券规则实施引起的信息不对称 问题。进一步的分析发现, 股权集中 度更高、内部控制更有效的公司在增 加企业社会责任行为方面的效应更为 显著。而且, 释放信号的激励越大增 加企业社会责任活动的效应越显著。 总之, 本文的研究结果显示公司经理
确实利用了企业社会责任活动作为证 明公司真实情况的信号机制。

需要说明的是在本文的分析中没 有体现公司应对融资融券规则实施到 底在企业社会责任活动中的哪些方面 做出了反应。因为润灵环球企业社会 责任评分是一个对公司社会责任行为 的综合衡量, 公司在企业社会责任相 关的任何一方面投资的变化, 都会体 现在企业社会责任总的评分之中。在 后续的研究中, 随着中国企业社会责 任有关数据的进一步细化、深化, 可 以使研究更进一步具体从而得到更有 针对性的结论。

\section{参考文献}

[1] Bénabou R,Tirole J.,"Individual and Corporate Social Responsibility"[J]. Economica. 2010, 305(77).

[2] Friedman M, "The Social Responsibility of Business is To Increase its Profits"[J]. The New York Times Magazine, 1970, 13(9): 122-126.

[3] Diether K B, Lee K H, Werner I M.,"Short-sale Strategies and Return Predictability"[J]. Review of Financial Studies. 2009, 22(2): 575607.

[4] 李志生,陈晨,林秉旋.卖空机制提高 了中国股票市场的定价效率吗？[J]. 经济研究, 2015 第 4 期.

[5] 陈国进,张贻军.异质信念、卖空限 制与我国股市的暴跌现象研究 $[\mathrm{J}]$. 金融研究, 2009 第 4 期.

[6] Goldstein I,E Ozdenoren, K Yuan., "Trading Frenzies and Their Impact on Real Investment"[J]. Journal of Financial Economics, 2013, 109(2): 566-582. 